\title{
Cytochrome $c$ /Multi-walled Carbon Nanotubes Modified Glassy Carbon Electrode for the Detection of Streptomycin in Pharmaceutical Samples
}

\author{
Rajasekhar ChOKKAREDDY, ${ }^{* \dagger}$ Gan G. REDHI, ${ }^{*}$ and Karthick ThANGAVEL ${ }^{* *}$ \\ *Department of Chemistry, Durban University of Technology, Durban-4000, South Africa \\ **Department of Physics, School of Electrical and Electronics Engineering, SASTRA Deemed University, \\ Thanjavur, 613401, Tamil Nadu, India
}

\begin{abstract}
A novel electrochemical glassy carbon electrode modified with a multi-walled carbon nanotube, cytochrome $c$ (Cyt $c$ ) and zinc oxide nanoparticles (ZnONPs) was fabricated to increase the sensitivity of electrode for the detection of streptomycin (STN) in certain pharmaceutical samples. Cyclic voltammetry (CV) and differential pulse voltammetry techniques were used for an electrochemical characterization of the electrode. Furthermore, the electrochemical biosensor construction phases were examined by using X-ray diffraction (XRD), transmission electron microscopy (TEM) and Fourier-transform infrared spectroscopy (FTIR). Under the optimal experimental conditions, the electrode offers a high selectivity and sensitivity signaling in the co-existence method of STN with the linear concentration ranging from 0.02 to $2.2 \mu \mathrm{M}$. The detection limits (LOD) and limit of quantification (LOQ) were found to be 0.0028 and $0.0562 \mu \mathrm{M}$, respectively. The fabricated sensing electrode has good stability, reproducibility and sensitivity towards STN in the pharmaceutical samples. Preliminary determinations of binding sites within the specified grid box size, which covers both Cyt $c$ and STN, were done by molecular docking analysis. Moreover, density functional theory (DFT) computations were performed to provide insightful information into the optimized geometry of STN.
\end{abstract}

Keywords Cytochrome $c$, streptomycin, different pulse voltammetry, molecular docking, DFT computations

(Received August 7, 2020; Accepted February 18, 2021; Advance Publication Released Online by J-STAGE March 5, 2021)

Transferable diseases are deadly enemies of the overall population and tuberculosis (TB) is among the highest of ten causes of global death. TB is an infectious disease caused by Mycobacterium tuberculosis. ${ }^{1-3}$ In 1882, the microbiologist Robert Koch described the tubercle bacillus, at a time when one of every seven deaths in Europe was affected by TB. In 2014, there was an estimated 9.6 million TB cases, including 5.4 millions of men, 3.2 millions of women and 1.0 million children. There were 1.5 million deaths (1.1 million among HIV-negative people and 0.4 million among HIV-positive people) reported due to TB. ${ }^{4-6}$ The number of deaths caused by TB is highly unacceptable. With a timely diagnosis and precise treatment, almost all TB patients can be cured. Streptomycin (STN) (Fig. 1) was the first antibiotic drug for TB. ${ }^{7}$ STN controls the bacterial growth by repairing cell membranes and inhibiting protein synthesis. Currently, STN is being extensively applied in infectious endocarditis, plague, and veterinary medicine to get rid of bacterial infectious diseases. Hence, its chemical synthesis and detection from biological fluids and pharmaceuticals are of utmost importance in the field of chemistry. In recent years, several techniques have been applied for STN detection in pharmaceuticals and biological fluids, such as high-performance liquid chromatography (HPLC), ${ }^{8}$ ionexchange-high-performance thin-layer chromatography (HPTLC), ${ }^{9,10}$ liquid chromatography-tandem mass spectrometry (LC-MS/MS), ${ }^{11}$ and colorimetric visualization. ${ }^{12}$ Although there

$\dagger$ To whom correspondence should be addressed.

E-mail: chokkareddys@gmail.com have been significant improvements in these techniques, their application is limited due to exclusive equipment and substances, a complicated wash process and time consuming. An electrochemical investigation is a rapid, powerful technique for identifying of such molecules due to its benefits of simple process, low cost, and high sensitivity in vivo determinations. Therefore, the detection of STN in biomaterials through electrochemical and other techniques ${ }^{7,13-16}$ are mainly attracted to researchers. However, there are some challenges while detecting STN in biomaterials due to its low accessibility, partial stability in temperature, $\mathrm{pH}$ dependence and biological solvents. ${ }^{17}$ As reported by Ghanbari and co-workers, a novel method employs gold nanoparticles and thiol graphene quantum dots as electrode modifiers for the determination of STN. The sensor showed a linear rage 0.1 to $700.00 \mathrm{pg} \mathrm{mL}^{-1}$, with a limit of detection (LOD) $0.033 \mathrm{pg} \mathrm{mL}^{-1} .^{18}$ Roushani and co-workers

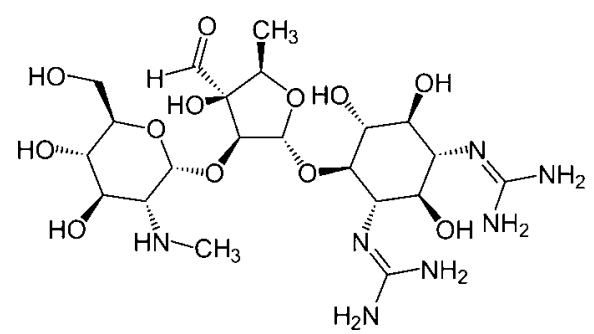

Fig. 1 Chemical structure of streptomycin. 
have developed an electrochemical aptasensor for detecting STN in milk and blood serum, by using a gold electrode fabricated with a thin mesoporous silica film and silver nanoparticles. The aptasensor shows a linear range $1 \mathrm{fg} \mathrm{mL}^{-1}$ to $6.2 \mathrm{ng} \mathrm{mL}^{-1}$, with a LOD of $0.33 \mathrm{fg} \mathrm{mL}^{-1} \cdot{ }^{19} \quad$ Zarei and co-workers have reported a novel sensor for determining STN in urine and plasma, by using a molecularly imprinted composite through the electro oxidation of 3-methyl-4-nitrophenol on GCE in the presence of $\mathrm{Au}(\mathrm{III})$. The proposed sensor shows a linear detection range of $0.35-4.5$ and $4.5-250 \mathrm{pM}$, with an LOD of 0.25 pM. $^{20}$ Akbarzadesh and co-workers have developed an electrochemical sensor for the detection of STN in the presence of oxytetracycline in milk samples. This determination exhibited two linear dynamic ranges, 0.4 to 240.0 and 240.0 to $720.0 \mathrm{nM}$, and a detection limit of $0.17 \mathrm{nM}$ for STN. ${ }^{21}$ However, the above described electrochemical sensors for STN determination were based on complex procedures for the synthesis of nanomaterials, and were often modified using multiple routes in plasma and urine samples only. In this investigation, we developed a novel senor for determining STN in pharmaceutical samples. Thus, novel and easier routes enabling the fabrication of electrochemical sensors procedures are highly desired for pharmaceutical samples. To overcome the above-mentioned challenges, an alternative sensing approach employing a hybrid material platform (like the one proposed in this work "Cyt $c$-ZnONPsMWCNTs-GCE") is essential. Metal oxide nanoparticles ( $\mathrm{ZnONPs}, \mathrm{TiO}_{2}$ and $\mathrm{Fe}_{3} \mathrm{O}_{4}$ ) are widely used in the sensor hybrid material platform due to their high catalytic activity and large surface area. $\mathrm{ZnONPs}, \mathrm{TiO}_{2}$ and $\mathrm{Fe}_{3} \mathrm{O}_{4}$ are semiconductors with respective band gap energy of $3.0,3.33$ and $3.36 \mathrm{eV}$ at room temperature. $^{22}$ The size and morphology of ZnONPs depends on the content of the precursors used in the synthesis of these NPs. ${ }^{23}$ Multi-walled carbon nanotubes (MWCNTs) are broadly used as an electrode coating material as they have a large specific surface area, electrochemical bond expansion, good adsorption properties, and they are chemically and thermally stable enough. ${ }^{22,24,25}$ Hence, they can be used to improve the sensitivity of the electrochemical response and making it appropriate for fastening the nanoparticles onto the outer surface of MWCNTs. Cytochrome $c$ (Cyt $c$ ) is a highly water-soluble hemeprotein found to loosely bind with the inner membrane of the mitochondrion. Cyt $c$ is an extremely conserved hemeprotein with a molecular weight of approximately $12 \mathrm{kDa}$. It consists of a single 104 amino acid peptide with a single heme group, which is attached to Cys ${ }^{14}$ and Cys ${ }^{17}$ through covalent bonds. ${ }^{26}$ The Cyt $c$ reacts with several organic and inorganic radicals ${ }^{27}$ and its unique electron transfer property can be used to construct an electrochemical biosensor. The Cyt $c$ carries an electron and is responsible for a redox mechanism in cell biology as well. Therefore, Cyt $c$ is also used as one of the electrode coating materials in our study. Finally, an effective Cyt $c$-based electrochemical biosensor was prepared on the surface of glassy carbon electrode (GCE) modified with MWCNTs and ZnONPs nanocomposite by an enzyme immobilization procedure. The electrochemical properties and analytical performance of the electrode were examined by cyclic voltammetry (CV) and differential pulse voltammetry (DPV) methods. The investigational parameters affecting the detectability of the sensor for STN were optimized in detail. The active binding sites between STN and Cyt $c$ were also predicted by molecular docking analysis. The local reactivity descriptors of STN were predicted using DFT calculations to obtain more structural insights. The analytical applications and selectivity performance of the sensing electrode were examined.

\section{Experimental}

\section{Reagents and chemicals}

The essential chemical supplements, such as sodium hydroxide, sulfuric acid, $N, N$-dimethyl formamide (DMF), disodium hydrogen phosphate, sodium dihydrogen phosphate, ethanol (99.9\%), zinc chloride, uric acid, glucose, sodium chloride, glutamic acid, folic acid, sucrose, potassium bromide, ferrous sulfate, nickel nitrate, potassium sulphate, potassium hydroxide and acetic acid, were obtained from Capital Lab Supplies (Durban, RSA). Pure analytical-grade of streptomycin and MWCNTs (outer diameter $7-15 \mathrm{~nm}$ and length $0.5-10 \mathrm{~m}$ ) was from Sigma Aldrich (Durban, RSA). Nitrogen gas with 99.9\% purity was supplied by AFROX (Durban, South Africa). Deionized water was produced from an aqua MAXTM-basic 360 series water distillation scheme, and was provided by Trilab Support (Durban, RSA). The alumina polishing cloth $(\leq 3 \mu \mathrm{M})$ was provided by Metrohm (Durban, RSA). All samples and reagents were prepared with deionized water.

\section{Preparation of $\mathrm{ZnONPs}$}

In order to synthesis $\mathrm{ZnONPs} \mathrm{nanoparticles,}{ }^{28} 5.45 \mathrm{~g}$ of $\mathrm{ZnCl}_{2}$ $(0.2 \mathrm{M})$ was dissolved in $200 \mathrm{~mL}$ ethanol and the resulting solution was constantly stirred for $30 \mathrm{~min}$ with the help of a magnetic stirrer. In addition, $1.12 \mathrm{~g}(0.4 \mathrm{M})$ of $\mathrm{KOH}$ was dissolved in $50 \mathrm{~mL}$ of ethanol to prepare an aqueous ethanol solution of $\mathrm{KOH}$. Then, a $0.4 \mathrm{M} \mathrm{KOH}$ solution was added dropwise without disturbing the solution much (allowed dropwise by touching the walls of the vessel) to a solution of completely dissolved $\mathrm{ZnCl}_{2}$ for $1 \mathrm{~h}$. The final solution was covered and allowed to settle down overnight. The precipitate of ZNONPs was separated carefully after extracting the supernatant solution and undergoing a centrifuging process of the remaining solution for $5 \mathrm{~min}$. Furthermore, the precipitate of $\mathrm{ZnONPs}$ were cleaned with double-deionized water three times, and then dried in an oven at $60^{\circ} \mathrm{C}$ for $1 \mathrm{~h}$, in order to convert the $\mathrm{Zn}(\mathrm{OH})_{2}$ into $\mathrm{ZnO}$.

\section{Fabrication of GCE with MWCNTs and ZnONPs}

A glassy carbon electrode was carefully polished with $0.3 \mu \mathrm{M}$ aluminium polishing cloth and rinsed with double-deionized water. Later, GCE electrode were washed with double-deionized water and dried at room temperature for $10 \mathrm{~min}$. Thereafter, $0.20 \mathrm{mg}$ of MWCNTs were dispersed in $5 \mathrm{~mL}$ of a DMF solution, and then the resulting mixture was sonicated for $60 \mathrm{~min}$ by using an ultra sonicator. In addition, $0.40 \mathrm{mg}$ ZnONPs and $0.40 \mathrm{mg}$ of MWCNTs were spread into $20 \mathrm{~mL}$ of DMF, and it obtain a gray suspension. Then, $10 \mu \mathrm{L}$ of $\mathrm{ZnONPs}-$ MWCNTs was drop casted onto the polished surface of a GCE electrode and kept for drying in an oven at $40^{\circ} \mathrm{C}$ for about $20 \mathrm{~min}$. The prepared ZnONPs-MWCNTs-GCE was then allowed to cool down to the room temperature, and finally, stored at $4^{\circ} \mathrm{C}$ in a refrigerator before use. In order to obtain an enzyme-immobilized biosensor, the ZnONPs-MWCNTs-GCE was dipped into the Cyt $c$ enzyme solution for $20 \mathrm{~min}$. Then, $30 \mu \mathrm{L}(0.6 \mathrm{mg} / \mathrm{mL})$ of Cyt $c$ was subsequently dropped onto the electrode surface for a 30 min incubation time. ${ }^{29}$ The electrode was then dried at ambient temperature for $15 \mathrm{~min}$ to obtain the proposed biosensor "Cyt $c$-ZnONPs-MWCNTs-GCE".

\section{Electrochemical measurements for biosensors}

First, $10 \mathrm{~mL} / 0.1 \mathrm{M}$ of a phosphate buffer solution (PBS) with $\mathrm{pH} 7$ was dropped onto an electrochemical cell in which the fabricated electrode was absorbed prior to the electrochemical 

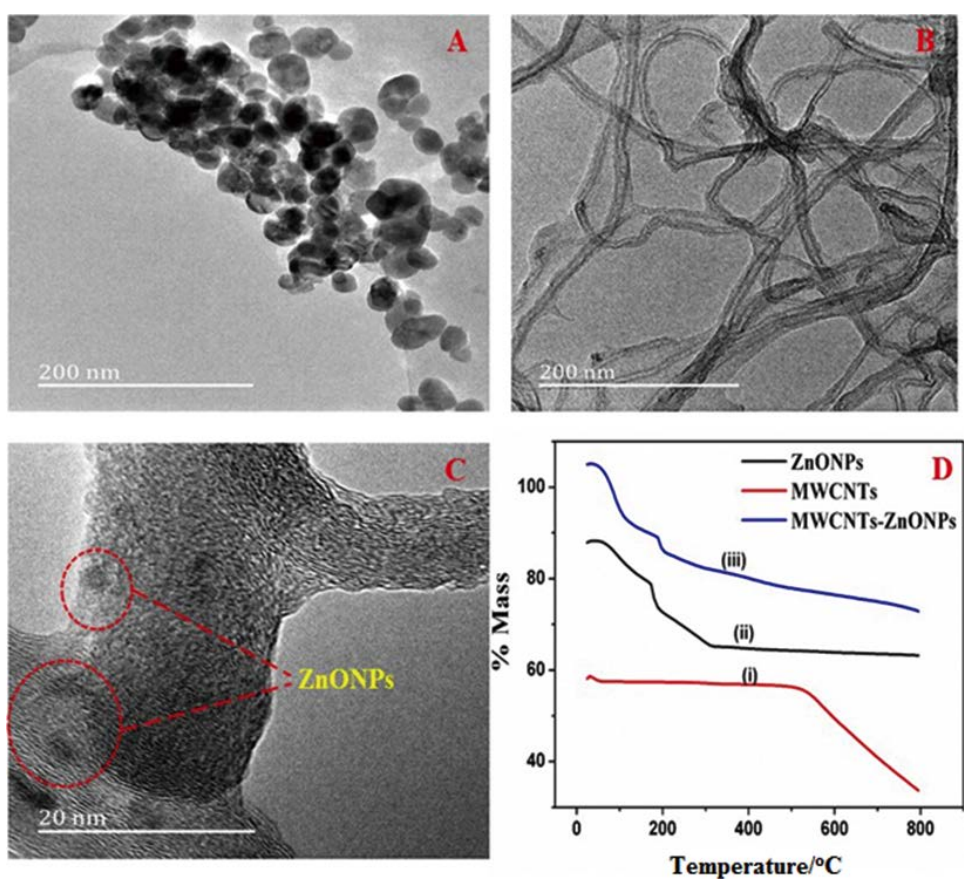

Fig. 2 TEM images for (A) ZnONPs (B) pure MWCNTs (C) ZnONPs decorated on MWCNTs (D) TGA curves for (i) MWCNTs (ii) ZnONPs (iii) MWCNTs-ZnONPs.

determinations. The electrochemical cell was purged with nitrogen gas for $15 \mathrm{~min}$ to remove dissolved oxygen. Then, the analyte was added into the cell and stirred at a speed of $1000 \mathrm{rpm}$. An aliquot of the analyte solutions was introduced into the electrochemical cell, and after the preconcentration time, stirring was stopped. An equilibration period of $10 \mathrm{~s}$ was allowed for the solution to become inert. The cyclic voltammetry and differential pulse voltammetry measurements were performed on the fabricated working electrode by scanning the potential towards the positive way, and sweeping was done at an optimized scan rate of $0.01 \mathrm{~V} \mathrm{~s}^{-1}$ by the DPV method. After each measurement, the working electrode was removed from the system and rinsed with deionized water.

\section{Streptomycin solution}

Streptomycin in commercially accessible formulation was admitted in the detection process. Tablet 1 specifies $300 \mathrm{mg}$ of streptomycin per tablet, Tablets 2 and 3 contain $500 \mathrm{mg}$ of streptomycin per tablet were taken for the preparation of pharmaceutical sample. The tablets were crushed with a mortar and pestle, and then the mass of the homogeneous powder was shifted into $25 \mathrm{~mL}$ volumetric flask. The powder samples were diluted with PBS ( $\mathrm{pH} 7)$. The obtained solution was ultrasonicated for $60 \mathrm{~min}$ in order to dissolve it completely and then filtered with Whatman Filter Paper No. 1. An appropriate aliquot amount of the solution was added to the supporting electrolyte in the voltammetric cell and analyzed directly by differential pulse voltammetry.

\section{Instrumentation}

Voltammetric measurements of the samples were carried out using a 797 VA analyzer with Computrace software Ver. 1.3.1 from Metrohm (Herisau, Switzerland). A three-electrode system consisting of a platinum wire (acting as a counter electrode), $\mathrm{Ag} / \mathrm{AgCl}$ (3.0 M KCl and acting as a reference electrode) and a fabricated GCE (acting as a working electrode). The solutions were filtered before the analysis using a $0.45 \mu \mathrm{M}$ pore size cellulose acetate filter medium and were examined by electrochemistry after the removal of impurities using nitrogen gas for 15 min. Differential Scanning Calorimetry (DSC) and Thermo Gravimetric Analysis (TGA) 1SF Model 1346 with STAR $^{\mathrm{e}}$ software Ver. 9.20 (Mettler Toledo, Columbus, OH, USA) instrument were used for the TGA characterization of the bio nanocomposite. The morphologies and characterizations were done with a TEM Model JEM 2100 equipped with a Lab6 emitter, Max Oxford instrument for TEM analysis (JEOL Inc., Peabody USA). Fourier-transform infrared (FT-IR) characterization was performed on a Varian 800 FT-IR scimitar series (by SMM instruments, Durban, RSA). The X-ray diffractions were performed with a Philips PW1710 X-Ray Diffraction Spectrometer (XRD). An ultrasonic bath (Labcon Model 5019 U) was used to prepare nanomaterial suspensions. A CRISON pH meter was used to prepare the buffer solutions. Deionized water was provided by the Aqua MAXTM-basic 360 system.

\section{Results and Discussion}

Morphological and structural characterization of Cyt c-ZnONPsMWCNTs-GCE

A TEM image of ZnONPs is shown in Fig. 2A. these figures confirm the formation of ZnONPs. Based on the Fig. 2A substantiate, the shape of ZnONPs was spherical. From the TEM image, the particle size is predicted to be approximately $7-12 \mathrm{~nm}$, which agrees with the XRD data. ${ }^{30,31}$ Figure 2B displays the TEM image of pure MWCNTs and it has tubularlike structure, with the nanotubes having clear inner channels. Figure $2 \mathrm{C}$ shows a TEM image of MWCNTs decorated with ZnONPs. It can be clearly seen that the $\mathrm{ZnO}$ nanoparticles are dispersed on the surface of MWCNTs. TGA studies of the ZnONPs, MWCNTs and ZnONPs-MWCNTs were performed from an ambient temperature to $800^{\circ} \mathrm{C}$. Figure $2 \mathrm{D}$ displays 


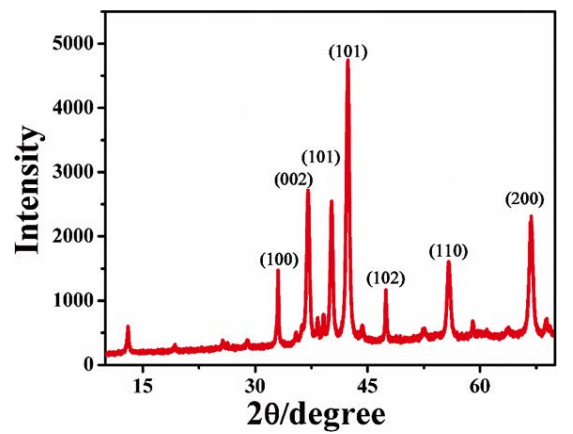

Fig. 3 XRD analysis of ZnONPs.

TGA curves for a usual precursor. At $190^{\circ} \mathrm{C}$, the ZnONPs loses a high amount of mass, which may due to the vaporization of water immersed on the surface. ${ }^{32}$ The trend arises continuously untill $380^{\circ} \mathrm{C}$ which specifies the high rate of degradation of ethyl alcohol into unstable flammable products. ${ }^{33}$ The thermograms for pure MWCNTs are revealed with a certain mass loss at $550^{\circ} \mathrm{C}$ due to the carbon oxidation (red line, Fig. 2D). Furthermore, the ZnONPs-MWCNTs demonstrates negligible weight loss below $100^{\circ} \mathrm{C}$ that can be attributed to the release of moisture. ${ }^{26}$ The produced $\mathrm{ZnONPs}$ were examined by the FT-IR method in order to confirm the synthesized ZnONPs. The IR spectrum of synthesized ZnONPs displays peaks at 450, $1070,1380,1602$ and $3453 \mathrm{~cm}^{-1}$. The sharp peak positioned at $450 \mathrm{~cm}^{-1}$ is attributed to the $\mathrm{Zn}-\mathrm{O}$ stretching bonds. ${ }^{34}$ In addition, the IR peaks at 2348 and $3453 \mathrm{~cm}^{-1}$ indicate the existence of the $\mathrm{C}=\mathrm{O}$ and $-\mathrm{OH}$ moieties. ${ }^{34,35}$ The IR peaks observed at 2947 and $2895 \mathrm{~cm}^{-1}$ are due to the symmetric and asymmetric $\mathrm{C}-\mathrm{H}$ bonds respectively. ${ }^{35}$ The XRD pattern of the ZnONPs is shown in Fig. 3. The strong diffraction peaks are positioned at $31.82^{\circ}(100), 37.25^{\circ}(002), 42.10^{\circ}(101), 43.44^{\circ}$ (101), $48.70^{\circ}(102), 57.41^{\circ}(110)$, and $63.29^{\circ}$ (200). Moreover, the peak strength is narrow and sharp, it supports good crystallinity of the ZnONPs. The unit cell of the crystal is found to be have a hexagonal structure. ${ }^{33,36}$ The particle size of ZnONPs is also calculated by using the Debye-Scherer formula,

$$
d=\frac{0.89 \lambda}{\beta \cos \theta},
$$

where 0.89 is Scherer's constant, $d$ is the average grain size, $\theta$ is the Braggs diffraction angle, $\lambda$ is the wavelength of $\mathrm{X}$-rays and $\beta$ is the full width at half maximum (FWHM) of the diffraction peak corresponding to the plane (101).

\section{Electrochemical characterization of the modified electrode}

The electrochemical response of STN at a bare electrode and modified electrodes in $0.1 \mathrm{M}$ PBS at $\mathrm{pH} 7$ was examined by cyclic and differntial pulse voltammetry. To observe the electrocatalytic performance of the Cyt $c$-ZnONPs-MWCNTsGCE towards the oxidation of STN, Fig. 4A represents the CV responses which were measured at $\mathrm{pH} 7 \mathrm{PBS}$ in the presence of STN. The anodic peak current intensity was increased, due to an increase in oxidation current density is the adsorption of STN in the solution to the surface of the fabricated GCE followed by conversion of Cyt $c$ (ox) to Cyt $c$ (red) reduced by STN. In addition, STN regenerates Cyt $c$ (red), an intense increase in the anodic current density appears at the potential scanning duration; moreover, the cathodic peak current intensity also increases while the STN is present (Fig. 4A). STN shows anodic and cathodic peaks in the potential window from -0.2 to $0.6 \mathrm{~V}$. Moreover, for all electrochemical assays with STN, the buffer solution was purged with nitrogen gas flow in order to remove any interference of the oxygen reduction reaction. Figure 4A shows cyclic voltammogramms of $0.1 \mathrm{mM}$ STN at the bare GCE (curve i), MWCNTs-GCE (curve ii), ZnONPs-MWCNTsGCE (curve iii) and Cyt $c$-ZnONPs-MWCNTs-GCE (curve iv) separately. It is worth mentioning that all of the modified electrodes show quasi-reversable redox peaks with various currents. The Cyt $c$-ZnONPs-MWCNTs-GCE $(185 \mu \mathrm{A})$ provides a greater electrochemical response than that of GCE $(12 \mu \mathrm{A})$, MWCNTs-GCE $(27 \mu \mathrm{A})$ and ZnONPs-MWCNTs-GCE $(92 \mu \mathrm{A})$, which might be sensibly attributed to the extraordinary electron transfer among the STN and electrode surface. The electrochemical active surface areas of the the bare GCE and Cyt $c$-ZnONPs-MWCNTs fabricated GCE can be calculated by the Randles-Ševčik equation. ${ }^{29}$

$$
i_{\mathrm{pa}}=2.69 \times 10^{5} A C_{0} n^{3 / 2} D_{\mathrm{R}}^{1 / 2} v^{1 / 2}
$$

where $C_{0}$ is the concentration of STN, $D_{\mathrm{R}}$ is the diffusion coefficient, $A$ is the surface area of the electrode, $i_{\mathrm{pa}}$ is the anodic peak current, $v$ is the scan rate and $n$ is the number of electrons transferred. $D_{\mathrm{R}}$ can be estimated from the oxidation peak current (Fig. 4A, curve i) of the bare GCE. The obtained $D_{\mathrm{R}}$ value may be used to estimate the surface area of the modified GCE. The fabricated sensor was found to have a higher surface area of $11.34 \mathrm{~mm}^{2}$ compared to that of bare GCE $\left(3.14 \mathrm{~mm}^{2}\right)$. Therefore, Cyt $c$-ZnONPs-MWCNTs-GCE was selected as the electrochemical biosensor for the studying electrochemical behavior of STN in following experiments.

\section{Influence $\mathrm{pH}$}

A performance of the working electrolyte as a biosensor in the determination of STN $(0.1 \mathrm{mM})$ is $\mathrm{pH}$ dependent. In the present study, an effect of $\mathrm{pH}$ in the range of 3 to 10 on the working electrolyte was examined by cyclic voltammetry and is presented in Fig. 4B. The peak-current intensity increases while increasing the $\mathrm{pH}$ values up to 7 , and the maximum intensity attains at $\mathrm{pH}$ 7. A further increase in the $\mathrm{pH}$ value does not increase the performance of working electrolyte, rather, its response decreases gradually. Figure $4 \mathrm{C}$ shows the variation of the electric potential with respect to the $\mathrm{pH}$. When the $\mathrm{pH}$ is decreased below 6 , the potential values become negative, which indicates the participation of the $\mathrm{H}^{+}$ions in the electrode reactions. Therefore, $\mathrm{PBS}$ with $\mathrm{pH} 7$ was considered to be the optimal $\mathrm{pH}$ and was used for subsequent experiments. The linear-regression equation was found to be $E_{\mathrm{P}}=0.0963 \mathrm{pH}+$ $0.483\left(R^{2}=0.9974\right)$. The resultant slope of the plot in the $\mathrm{pH}$ range from 3 to 10 demonstrates that the numbers of electrons and protons are equal in the electrochemical redox reaction of STN, as previously reported. ${ }^{37}$

\section{Deposition time}

Due to the adsorptive nature of STN at the electrode surface, cyclic and differential pulse voltammetry methods were selected as a sensitive technique for the detection of STN. The deposition of STN rapidly increases the electrochemical response. The effect of the deposition time on the CV signal of $0.1 \mathrm{mM}$ STN was examined. With increasing deposition time from 30 to $90 \mathrm{~s}$, the peak currents are gradually increased, as shown in Fig. 4D. The peak current reaches its maximum at a deposition time of $90 \mathrm{~s}$, after that peak current starts decreasing due to the saturation of the electrode surface. Therefore, $90 \mathrm{~s}$ was chosen as the optimized deposition time for the entire study. 

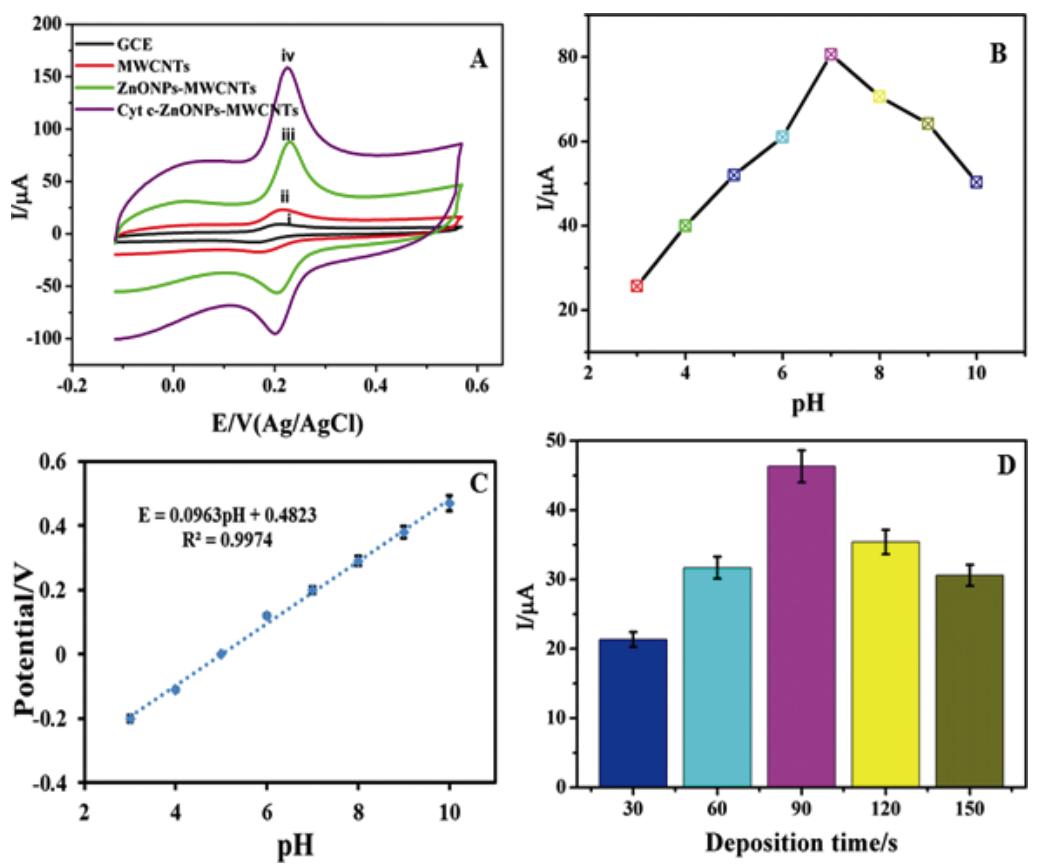

Fig. 4 (A) Cyclic voltammograms of STN (0.1 mM) at bare GCE (i), MWCNTs-GCE (ii), ZnONPsMWCNTs-GCE (iii) and Cyt $c$-ZnONPs-MWCNTs-GCE (iv) in 0.1 M PBS (pH 7.0). (B) Cyclic voltammograms of STN $(0.1 \mathrm{mM})$ in different $\mathrm{pH}$ of solutions: (3.0, 4.0, 5.0, 6.0, 7.0, 8.0, 9.0 and $10.0)$. (C) Plot of the peak potential $\left(E_{\mathrm{p}}\right)$ of anodic wave versus $\mathrm{pH}(\mathrm{pH} 3-10)$. (D) Various deposition times $(30,60,90,120$, and $150 \mathrm{~s})$ on peak current.

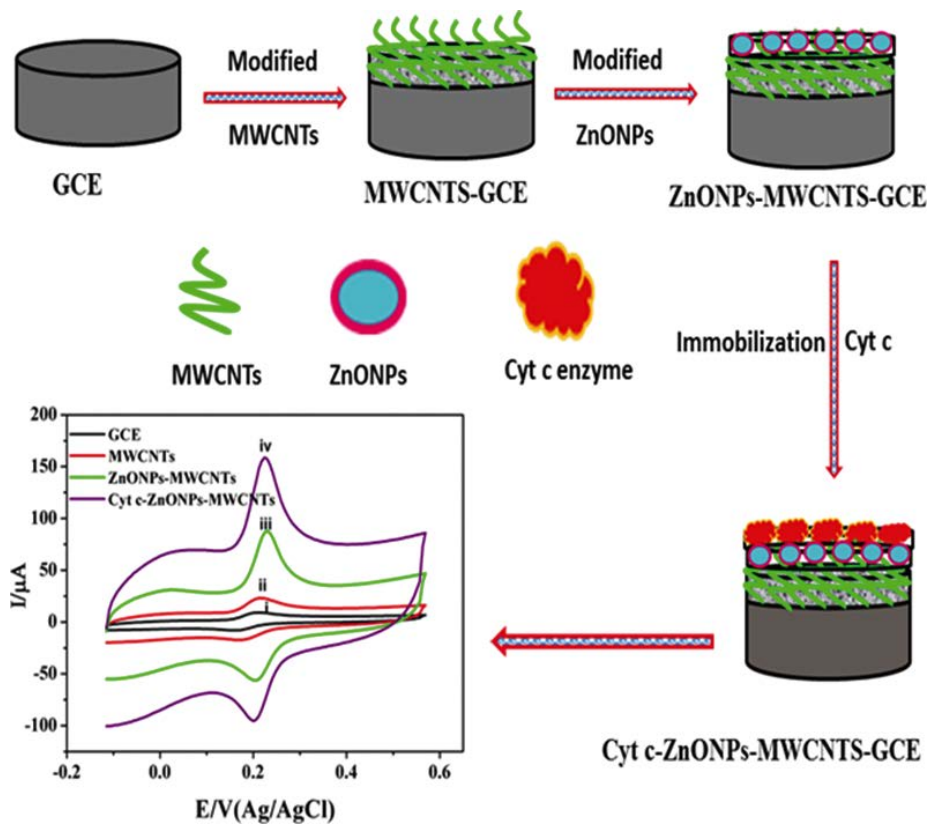

Scheme 1 Schematic illustration of the STN electrochemical biosensor based on Cyt $c$-ZnONPsMWCNTs nanocomposite.

Sensing mechanism and influence of scan rates variation

In this investigation, the electrochemical biosensor was modified by covalently attaching Cyt $c$ to the MWCNTs and ZnONPs coated on a glassy carbon electrode surface. The MWCNTs and ZnONPs act as carriers of the electrochemical capture probe to increase the change of the peak currents. In addition to that, Cyt $c$ is used as a redox mediator to generate the electron flow between STN and modified electrode (Scheme 1). A result of the possible scan rate on the electrochemical properties of Cyt $c$-ZnONPs-MWCNTs-GCE is investigated in $0.1 \mathrm{mM}$ PBS $(\mathrm{pH})$ by using the CV (see Fig. 5A). Initially, the redox peaks of STN increases steadily with increasing scan rates from 0.01 to $0.1 \mathrm{mV} \mathrm{s}^{-1}$ within the potential range of -0.2 to $0.6 \mathrm{~V}$. It was observed that there is a linear relationship 

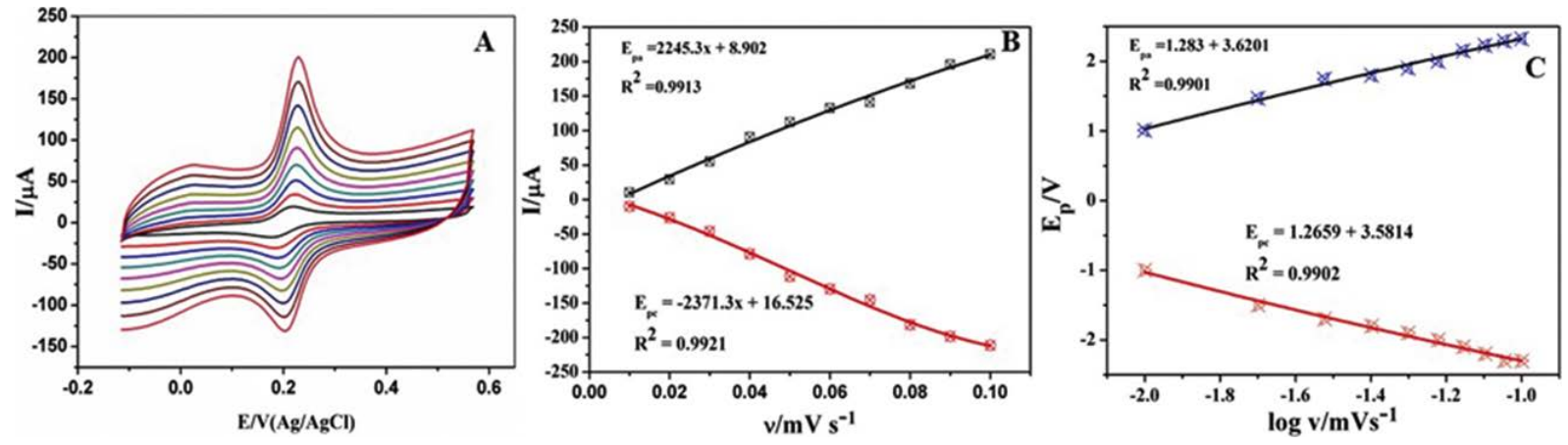

Fig. 5 (A) CV responses of the Cyt $c$-ZnONPs-MWCNTs-GCE in PBS buffer solution (pH 7) at scan rates (inner to outer) $0.01,0.02,0.03,0.04,0.05,0.06,0.07,0.08,0.09$ and $0.1 \mathrm{mV} \mathrm{s}^{-1}$. (B) Plot of anodic and cathodic peak current $v s$. scan rate $(\mathrm{C})$ is the variation of anodic and cathodic potential $v s$. logarithm of scan rate.

between the peak currents and scan rates. Figure 5B shows the dependence of the anodic and cathodic peaks with respect to the scan rates on the solution of STN. A linear dependency of the redox reaction up on the scan rate $(v)$ was observed, which demonstrates an adsorption-controlled procedure. In order to confirm this result, a plot between the logarithmic values of peak currents and logarithmic values of scan rates is plotted as shown in Fig. 5C. It provides straight lines with slopes of 1.283 and 1.269 for cathodic and anodic peak currents, individually. The slope values are the same and close to the theoretical value of an ideal adsorption controlled electrochemical reaction (1.12). While increasing the scan rates, the anodic peak potential $\left(E_{\mathrm{pa}}\right)$ moves to the positive direction and the cathodic peak potential $\left(E_{\mathrm{pc}}\right)$ moves to the negative direction. Therefore, the peak to peak separation $\left(E_{\mathrm{p}}\right)$ was somewhat increased, this behavior demonstrates the quasi-reversible electrochemical process. In order to determine the electrochemical limitations, the difference of the peak potentials with the logarithm of the scan rates at a high scan rate was further plotted according to the Laviron Eq. (4). ${ }^{38}$

$$
\begin{aligned}
& E_{\mathrm{pa}}=E^{0}-\frac{2.3 R T}{\alpha n F} \log v \\
& E_{\mathrm{pc}}=E^{0}-\frac{2.3 R T}{(1-\alpha) n F} \log v
\end{aligned}
$$

$$
\begin{aligned}
\log k_{\mathrm{s}}=\alpha \log (1-\alpha)+(1-\alpha)- & \log \left(\frac{R T}{n F v}\right) \\
& -\alpha(1-\alpha)\left(\frac{n F \Delta E_{\mathrm{p}}}{2.3 R T}\right)
\end{aligned}
$$

where $\alpha$ is the electron transfer coefficient, $k_{\mathrm{s}}$ is the apparent electron transfer rate constant, $\mathrm{n}$ is the number of electron transfer, $R$ is the universal gas constant, $T$ is the temperature and $E_{\mathrm{p}}$ is the peak to peak separation, i.e. $E_{\mathrm{pa}}-E_{\mathrm{pc}}$. The fabrication procedure for the proposed electrochemical biosensor is illustrated in Scheme 1.

\section{Optimization of incubation time of Cyt c}

In order to optimize the enzyme, the incubation time is one of the most significant parameters. For determining of the STN, it was studied from 5 to $20 \mathrm{~min}$ at ambient temperature as shown in Fig. 6. It was initially found that various incubation times of STN produce a visible difference in the peak currents. The

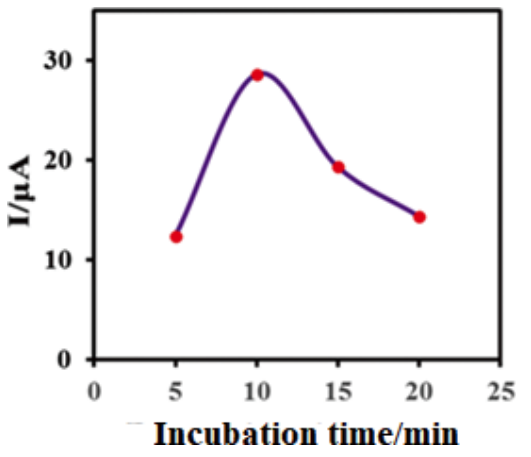

Fig. 6 Effect of the incubation time on the response of Cyt $c$ ZnONPs-MWCNTs-GCE.

results show that the peak current response steadily amplified with extended incubation time and achieved equilibrium at $10 \mathrm{~min}$. After $10 \mathrm{~min}$, the peak currents slowly decreases due to the saturation of the electrode surface. Therefore, 10 min was chosen as the incubation time for the determination of STN.

\section{Reproducibility and stability of the biosensor}

To investigate the applicability of the proposed electrode, the repeatability of STN detection, stability and reproducibility were studied under the optimized experimental conditions. In order to examine the reproducibility of the electrode preparation process, six modified electrodes constructed on the same fabrication procedure and used for the detection of $0.1 \mathrm{mM}$ STN solution by the DPV technique. From six similar detections, the relative standard deviation (RSD) for the determination of STN was calculated, and found to be $2.86 \%$, which shows a good sensor-to-sensor reproducibility. Furthermore, the repeatability of the anticipated biosensor was examined by detection of $0.1 \mathrm{mM}$ STN and variation coefficient of $3.12 \%$ was observed for six successive assays. The peakcurrent response of the Cyt $c$-ZnONPs-MWCNTs-GCE was unchanged from its initial value of $96.8 \%$ after 10 successive assays (RSD $2.21 \%$ ). Furthermore, the stability of the modified electrode was investigated by its voltammetric response on the peak current to $0.1 \mathrm{mM}$ STN over 30 days. In the first 15 days, the action of the sensor was engaged about $93 \%$ of its original peak current response. Moreover, after 15 days, its response decreased slowly. The Cyt $c$-ZnONPs-MWCNTs-GCE showed $89 \%$ of the initial performance at the end of the 30th day. 


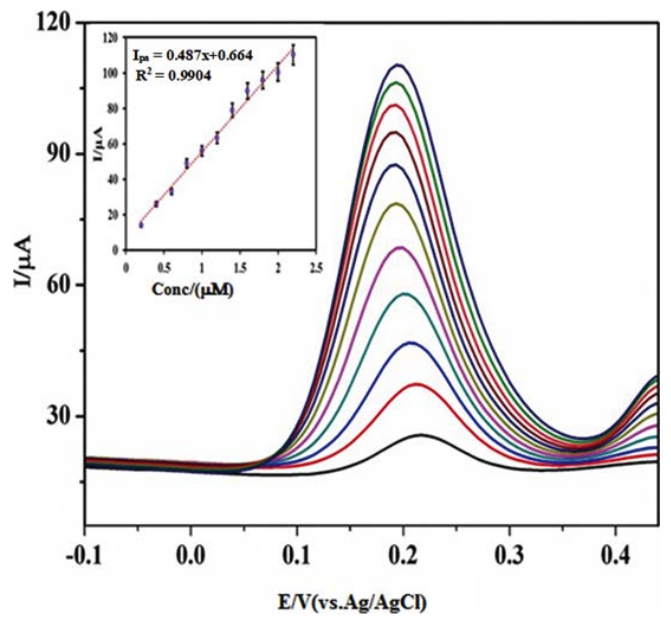

Fig. 7 DPV curves recorded on Cyt $c$-ZnONPs-MWCNTs-GCE with the successive addition of STN from 0.2 to $2.2 \mu \mathrm{M}$. Insert the calibration plot of the concentration of STN peak current on Cyt $c$-ZnONPs-MWCNTs-GCE.

This indicates that the proposed electrochemical sensor has good stability over a period of months. Shen and co-workers has reported a photoelectrochemical and electrochemical ratiometric aptasensing for STN. ${ }^{39}$ The Cyt $c$-ZnONPs-MWCNTs-GCE biosensor showed a low LOD, good stability and satisfactory reproducibility results, compared to the above-mentioned photoelectrochemical and electrochemical ratiometric sensor.

\section{Calibration curve for electrochemical detection of STN}

Due to the higher peak current sensitivity and superior peak separation, DPV was used for the sensitive detection of STN using an enzyme immobilized biosensor Cyt $c$-ZnONPsMWCNTs-GCE. The optimal experimental conditions (accumulation potential $0.18 \mathrm{~V}$, accumulation time $60 \mathrm{~s}$, voltage step time $0.38 \mathrm{~s}$, pulse amplitude $0.051 \mathrm{~V}$, scan rate $0.012 \mathrm{~V} \mathrm{~s}^{-1}$, voltage step $0.21 \mathrm{~V}, 0.1 \mathrm{M}$ PBS and $\mathrm{pH} 7$ ) were maintained while performing the experiment. Furthermore, a sequence of DPVs was noted at several concentrations of STN to define its calibration curve. The reaction of the Cyt $c$-ZnONPs-MWCNTsGCE to STN were found to be increased while increasing the concentration of STN. Figure 7 displays DPV curves recorded on the Cyt $c$-ZnONPs-MWCNTs-GCE at different STN concentrations in the range of 0.02 to $2.2 \mu \mathrm{M}$. Repetitive measurements exposed the possible passivation of the electrode by the products of electrode adsorption or reaction of the STN onto the electrode surface. This results in flowing of the peaks to more positive potentials. The linear regression equation was $I_{\mathrm{pa}}(\mu \mathrm{A})=0.487 x+0.664\left(R^{2}=0.9904\right)$ with $0.0028 \mu \mathrm{M}$ of LOD and $0.0562 \mu \mathrm{M}$ of LOQ at the signal to noise ratio of 3 . Therefore, this electrochemical biosensor can be used successfully for the determination of STN in pharmaceutical samples.

\section{Interference studies}

The selectivity of Cyt $c$-ZnONPs-MWCNTs-GCE for the determination of STN in the mixer of certain inorganic salts and biological substances were studied. The developed biosensor is allowed to enter into the mixer of $0.1 \mu \mathrm{M}$ STN with the interference substances individually with various concentrations for competing the reactions for $15 \mathrm{~min}$. The differential pulse voltammetry is used to detect the interfering ions in $10 \mathrm{~mL}$ of $0.1 \mathrm{mM}$ PBS at $\mathrm{pH}$ 7. The concentrations of various interferences
Table 1 Influences of some inorganic salts and important biological substances on the peak current of $0.1 \mathrm{mM}$ streptomycin at Cyt $c$-ZnONPs-MWCNTs-GCE

\begin{tabular}{lcc}
\hline \multicolumn{1}{c}{ Interferent } & Concentration $/ \mu \mathrm{M}$ & Signal change, $\%$ \\
\hline $\mathrm{NaCl}$ & 500 & 1.88 \\
$\mathrm{AgNO}_{3}$ & 500 & 1.95 \\
$\mathrm{CaCl}_{2}$ & 250 & 0.93 \\
$\mathrm{NaSO}_{4}$ & 500 & 1.86 \\
Sucrose & 500 & 0.25 \\
Glucose & 500 & 0.29 \\
Urea & 250 & 2.2 \\
Ascorbic acid & 250 & 3.89 \\
Dopamine & 250 & 1.20 \\
Citric acid & 250 & 1.61 \\
\hline
\end{tabular}

Table 2 Determination of STN in various pharmaceutical samples using Cyt $c$-ZnONPs-MWCNTs-GCE $(n=5)$

\begin{tabular}{ccccc}
\hline $\begin{array}{c}\text { Tablet } \\
\text { sample }\end{array}$ & $\begin{array}{c}\text { Spiked/ } \\
\mu \mathrm{M}\end{array}$ & $\begin{array}{c}\text { Observed/ } \\
\mu \mathrm{M}\end{array}$ & $\begin{array}{c}\text { Recovery, } \% \\
(n=3)\end{array}$ & $\begin{array}{c}\text { RSD, \% } \\
(n=5)\end{array}$ \\
\hline Tablet 1 & 5 & 4.93 & 98.6 & 1.22 \\
& 10 & 9.50 & 95.0 & 0.75 \\
& 15 & 14.85 & 99.0 & 1.41 \\
Tablet 2 & 20 & 20.12 & 100.6 & 2.11 \\
& 5 & 5.02 & 100.4 & 2.31 \\
& 10 & 9.89 & 98.9 & 1.69 \\
Tablet 3 & 15 & 14.89 & 99 & 1.95 \\
& 20 & 19.87 & 99.3 & 3.12 \\
& 5 & 4.86 & 97.2 & 1.57 \\
& 10 & 10.12 & 101.2 & 2.09 \\
& 15 & 15.21 & 101.4 & 1.78 \\
& 20 & 21.01 & 105 & 1.82 \\
\hline
\end{tabular}

substances and their signal changes are listed in Table 1. Moreover, the tolerance limit was defined as the maximum concentration of interference substances, which can cause an error $\pm 4 \%$ in the determination of STN. These effects indicate that the suggested biosensor possesses a good anti-interference capability for the detection of STN.

\section{Analytical application for the proposed sensor}

As related to the linear regression equations of bulk form, the developed sensor was successfully utilized for the quantification of STN in tablet dosage forms without any matrix effects. The real sample preparation process is described in the experimental section. For a real sample investigation, an appropiate amount was directly dissloved in deionised water, followed by serial dilution of a $10 \mathrm{~mL}$ phosphate buffer solution ( $\mathrm{pH} 7)$. In addition, recovery studies were employed by the standard addition method, that a known concentrations of pure STN was added to the tablet dosage form. Each sample was tested five times and the detected value was the average of five measurements. The recovery parameters were calculated using the related calibration equations. The recovery was in the range 95 to $105 \%$, and the results are precised and recorded in Table 2. It can be concluded that the found $\mathrm{RSD} \%$ of recovery values prove that the developed electrochemical biosensor has considerably great potential for the consistency and sensitivity to the detection of STN in pharmaceutical samples.

\section{Reactivity descriptors of STN}

The detection of STN using biosensors requires a basic 
Table 3 The atomic sites that are prone to electrophilic and nucleophilic attack of STN

\begin{tabular}{|c|c|c|c|c|c|c|c|c|}
\hline \multirow{2}{*}{ Atom No. } & \multicolumn{3}{|c|}{ Hirshfeld charge } & \multicolumn{3}{|c|}{ Fukui function } & \multicolumn{2}{|c|}{ Relative electrophilicity/nucelophilicity } \\
\hline & $\mathrm{N}$ & $\mathrm{N}-1$ & $\mathrm{~N}+1$ & $f_{\mathrm{k}}^{+}$ & $f_{\mathrm{k}}^{-}$ & $f_{\mathrm{k}}^{0}$ & $f_{\mathrm{k}}^{+} / f_{\mathrm{k}}^{-}$ & $f_{\mathrm{k}}-/ f_{\mathrm{k}}^{+}$ \\
\hline O5 & -0.0609 & -0.0954 & -0.0538 & 0.0345 & 0.0071 & 0.0208 & 4.8524 & 0.2061 \\
\hline O6 & -0.1238 & -0.1264 & -0.1083 & 0.0026 & 0.0155 & 0.0091 & 0.1683 & 5.9407 \\
\hline O11 & -0.2068 & -0.3393 & -0.1928 & 0.1325 & 0.0139 & 0.0732 & 9.5215 & 0.1050 \\
\hline N13 & -0.2217 & -0.2249 & -0.1803 & 0.0033 & 0.0414 & 0.0223 & 0.0788 & 12.6854 \\
\hline N14 & -0.2272 & -0.2314 & -0.1134 & 0.0042 & 0.1139 & 0.0590 & 0.0370 & 26.9919 \\
\hline $\mathrm{C} 20$ & 0.0790 & 0.0606 & 0.0798 & 0.0184 & 0.0008 & 0.0096 & 22.6900 & 0.0441 \\
\hline $\mathrm{C} 22$ & 0.0636 & 0.0534 & 0.0644 & 0.0102 & 0.0009 & 0.0055 & 11.5619 & 0.0865 \\
\hline $\mathrm{C} 23$ & 0.1407 & 0.1261 & 0.1417 & 0.0146 & 0.0010 & 0.0078 & 15.3270 & 0.0652 \\
\hline $\mathrm{C} 25$ & 0.0609 & 0.0608 & 0.0718 & 0.0001 & 0.0109 & 0.0055 & 0.0085 & 117.9565 \\
\hline $\mathrm{C} 27$ & 0.0792 & 0.0540 & 0.0811 & 0.0251 & 0.0020 & 0.0136 & 12.6985 & 0.0787 \\
\hline C29 & 0.0276 & 0.0258 & 0.0555 & 0.0019 & 0.0279 & 0.0149 & 0.0672 & 14.8715 \\
\hline $\mathrm{C} 35$ & 0.0276 & -0.0295 & 0.0418 & 0.0570 & 0.0142 & 0.0356 & 4.0121 & 0.2492 \\
\hline C36 & 0.1950 & -0.0158 & 0.1981 & 0.2108 & 0.0031 & 0.1070 & 67.0108 & 0.0149 \\
\hline C38 & 0.1372 & 0.1351 & 0.1598 & 0.0021 & 0.0226 & 0.0123 & 0.0926 & 10.7988 \\
\hline
\end{tabular}

Table 4 Local reactivity descriptors of STN

\begin{tabular}{cccccccc}
\hline$\varepsilon_{\mathrm{H}} / \mathrm{eV}$ & $\varepsilon_{\mathrm{L}} / \mathrm{eV}$ & $\varepsilon_{\mathrm{H}}-\varepsilon_{\mathrm{L}} / \mathrm{eV}$ & $X$ & $\mu$ & $\eta$ & $W_{\text {max }}$ & \\
\hline-5.9881 & -2.1214 & 3.8667 & 4.0548 & -4.0548 & 1.9334 & 0.2586 & 4.2519 \\
\hline
\end{tabular}

understanding of reactive sites on the STN for electrophilic and nucleophilic reactions. For this purpose, the descriptors (local and global reactivity) used to evaluate the reactivity of the molecule, were predicted by DFT calculations at the B3LYP/6$311++\mathrm{G}(\mathrm{d}, \mathrm{p})$ level. Fukui functions corresponding to anionic, cationic and neutral radical species of STN were estimated by the folowing equations. ${ }^{40,41}$ We incorporated the hirshfeld charges instead of the Mulliken charges for a better accuracy of the results and these results were compared with the electrostatic potential surface (ESP). ${ }^{42}$

$$
\begin{aligned}
& f_{\mathrm{A}}^{\text {neu }}=\frac{1}{2}\left[q_{\mathrm{A}(\mathrm{N}-1)}-q_{\mathrm{A}(\mathrm{N}+1)}\right] \\
& f_{\mathrm{A}}^{\text {cation }}=\frac{1}{2}\left[q_{\mathrm{A}(\mathrm{N})}-q_{\mathrm{A}(\mathrm{N}+1)}\right] \\
& f_{\mathrm{A}}^{\text {anion }}=\frac{1}{2}\left[q_{\mathrm{A}(\mathrm{N}-1)}-q_{\mathrm{A}(\mathrm{N})}\right]
\end{aligned}
$$

Where $f_{\mathrm{A}}^{\text {neu }}, f_{\mathrm{A}}^{\text {cation }}$ and $f_{\mathrm{A}}^{\text {anion }}$ are the Fukui functions confirming to the neutral radical, nucleophilic and electrophilic reactions, individually. Mainly, the nucleophiles and electrophiles involve in making interactions with the residues of protein/enzyme. The atomic sites of STN that are prone to the electrophilic/ nuceleophilic attack are depicted in Table 3. The carbon and oxygen atoms in the aldehyde group that aquire a higher $f_{\mathrm{k}}{ }^{+} / f_{\mathrm{k}}{ }^{-}$, and are prone to be attacked by the nucleophile. The linkers (C-O) that connect the six and five membered rings are prone to be attacked by the nucleophile.

The highest nuecleophilicity index acquired by the atoms $(\mathrm{C} 25, \mathrm{C} 29, \mathrm{~N} 13$ and N14) are prone to electrophilic attack. Moreover, the global reactivity descriptors of STN, such as HOMO-LUMO energy gap $\left(\varepsilon_{\mathrm{H}}-\varepsilon_{\mathrm{L}}\right)$, ionization potential $(I)$, electron affinity $(A)$, electronegativity $(\chi)$, chemical potential $(\mu)$, global hardness $(\eta)$, global softness (S), and global electrophilicity index $(\omega)$ were calculated (Table 4$)$. It shows a very small energy gap of $3.8667 \mathrm{eV}$. Thus, the molecule STN is

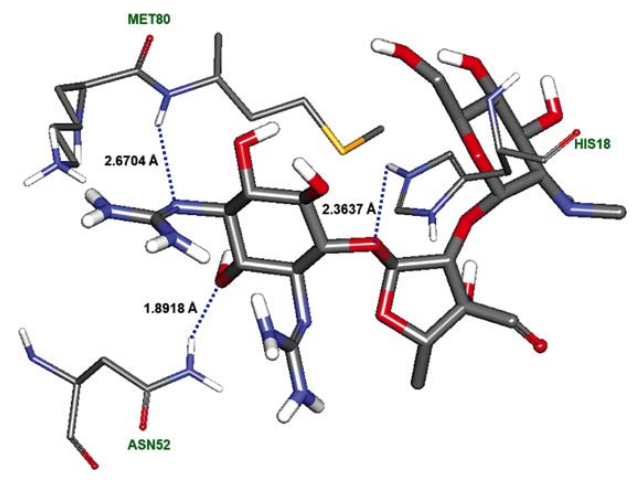

Fig. 8 Lowest energy docked conformation of STN and Cyt $c$.

more reactive and the atomic sites that are predicted using the Fukui functions are helpful to understand the chemical reactivity of the molecule.

\section{Active binding sites between STN and Cyt c}

The proposed sensor shows a significant sensing ability of STN in pharmaceutical substances and in mixers containing inorganic salts, the binding sites between the STN and Cyt $c$ (pdb code: $3 \mathrm{NWV}$ ) are of future scope to this work. The energy minimization of 10 docked conformations were performed by Lamarcian Genetic Algorithm (LGA) in the Autodock suit of programs. Standard grid dimensions of $60 \times 60 \times 60$ were fixed in order to assemble the ligand and the target in the search space. Figure 8 visualizes the lowest energy docked conformation of STN and Cyt $c$. The docked conformation illustrates that STN makes strong binding with the residues ASN52, HIS18 and MET80 through hydrogen bonds. The binding energy of the lowest energy conformation is estimated to be $1.87 \mathrm{kcal} / \mathrm{mol}$ with the highest torsional energy of 4.77 $\mathrm{kcal} / \mathrm{mol}$. 


\section{Conclusions}

The electrochemical behavior of STN was examined with appropriate techniques. STN was found to be electrochemically energetic in the $\mathrm{pH}$ range of $3.0-10.0$, but the peak currents of STN were higher at $\mathrm{pH}$ 7. GCE was fabricated with MWCNTs, which enhanced the response of STN noticeably. Furthermore, the addition of ZnONPs to the MWCNTs to form a nanocomposite electrode, and then immobilizing composite electrode with Cyt $c$, resulted in the electrochemical biosensor. The higher peak currents, and the response of the STN were appreciably increased and were explained in detail. The fabrication of such electrochemical sensor immobilized with biomolecules can fruitfully be used for analytical applications of STN with simplicity, time saving and reduced costs. The determination of STN was proposed synchronously with varied linear ranges from 0.02 to $2.2 \mu \mathrm{M}$, and the LOD, LOQ was obtain to be 0.0028 and $0.0562 \mu \mathrm{M}$, respectively $(S / N=5)$. After the experimental limitations affecting the response sensitivity of the biosensor were optimized, the resulting biosensor not only exhibited high sensitivity and selectivity of STN, but also showed much better reproducibility and repeatability.

\section{Acknowledgements}

The authors gratefully acknowledge Durban University of Technology, Durban, South Africa for financial assistance. K. T. acknowledges SASTRA Deemed University, the management of SASTRA Deemed University, Thanjavur, Tamil Nadu, India for providing necessary infrastructure and computational facilities.

\section{References}

1. WHO, "The World Health Report 2000: Health Systems: Improving Performance", 2000, World Health Organization.

2. R. Chokkareddy, N. Bhajanthri, G. G. Redhi, and D. G. Redhi, Curr. Anal. Chem., 2018, 14, 391.

3. R. Chokkareddy, N. Bhajanthri, and G. Redhi, Biosensors, 2017, 7, 21.

4. J. C. Palomino, S. C. Leão, and V. Ritacco, "Tuberculosis 2007: From Basic Science to Patient Care”, 2007, 1.

5. M. S. Vasava, M. N. Bhoi, S. K. Rathwa, M. A. Borad, S. G. Nair, and H. D. Patel, Indian J. Tuberc., 2017, 64, 252.

6. R. Chokkareddy, Doctoral Dissertation, 2018.

7. X. Que, B. Liu, L. Fu, J. Zhuang, G. Chen, and D. Tang, Electroanalysis, 2013, 25, 531.

8. B. Chen, H. Zhang, B. Lin, J. Ge, and L. Qiu, J. AOAC Int., 2012, 95, 523.

9. V. Ghoulipour, M. Shokri, and S. Waqif-Husain, J. Planar Chromatogr.-Mod. TLC, 2011, 24, 520.
10. H. Abbasi and K.-E. Hellenäs, Analyst, 1998, 123, 2725.

11. M. Preu and M. Petz, J. Chromatogr. A, 1999, 840, 81.

12. J. Sun, J. Ge, W. Liu, Z. Fan, H. Zhang, and P. Wang, Chem. Commun., 2011, 47, 9888.

13. S. M. Taghdisi, N. M. Danesh, M. A. Nameghi, M. Ramezani, and K. Abnous, Food Chem., 2016, 203, 145.

14. V. Soheili, S. M. Taghdisi, M. H. Khayyat, B. S. F. Bazzaz, M. Ramezani, and K. Abnous, Microchim. Acta, 2016, 183, 1687.

15. G. K. Mishra, A. Sharma, and S. Bhand, Biosens. Bioelectron., 2015, 67, 532.

16. A. S. Emrani, N. M. Danesh, P. Lavaee, M. Ramezani, K. Abnous, and S. M. Taghdisi, Food Chem., 2016, 190, 115.

17. C. Rao and G. Reddi, TrAC Trends Anal. Chem., 2000, 19, 565.

18. S. Akbarzadeh, H. Khajesharifi, and M. Thompson, Biosensors, 2020, 10, 23.

19. M. Roushani and K. Ghanbari, Microchim. Acta, 2019, 186, 1.

20. K. Zarei and M. Ghorbani, Electrochim. Acta, 2019, 299, 330.

21. K. Ghanbari and M. Roushani, Bioelectrochemistry, 2018, 120,43

22. R. Chokkareddy, N. K. Bhajanthri, and G. G. Redhi, Int. J. Electrochem. Sci., 2017, 12, 9190.

23. R. Chokkareddy, N. K. Bhajanthri, and G. G. Redhi, Indian J. Chem., Sect. A, 2018, 57, 887 .

24. N. K. Bhajanthri, V. K. Arumugam, R. Chokkareddy, and G. G. Redhi, J. Mol. Liq., 2016, 222, 370.

25. R. Chokkareddy, G. G. Redhi, and T. Karthick, Heliyon, 2019, 5, e01457.

26. Y. Hatefi, Annu. Rev. Biochem., 1985, 54, 1015.

27. W. Koppenol and J. Butler, Isr. J. Chem., 1984, 24, 11.

28. N. S. Rao and M. V. B. Rao, Am. J. Mater. Sci., 2015, 5, 66.

29. J. C. Kilele, R. Chokkareddy, N. Rono, and G. G. Redhi, J. Taiwan Inst. Chem. Eng., 2020, 111, 228.

30. M. AbdElhady, Int. J. Carbohydr. Chem., 2012, 2012, 1.

31. P. Taunk, R. Das, D. Bisen, and R. Kumar Tamrakar, J. Radiat. Res. Appl. Sci., 2015, 8, 433.

32. M. Mohamed, W. Salleh, J. Jaafar, and A. Ismail, J. Sol-Gel Sci. Technol., 2015, 74, 513.

33. M. I. Khalil, M. M. Al-Qunaibit, A. M. Al-Zahem, and J. P. Labis, Arab. J. Chem., 2014, 7, 1178.

34. A. Becheri, M. Dürr, P. Lo Nostro, and P. Baglioni, J. Nanopart. Res., 2008, 10, 679.

35. A. K. Zak, W. A. Majid, M. Darroudi, and R. Yousefi, Mater. Lett., 2011, 65, 70.

36. S. Talam, S. R. Karumuri, and N. Gunnam, ISRN Nanotechnology, 2012, 2012, 1.

37. B. Liu, D. Tang, B. Zhang, X. Que, H. Yang, and G. Chen, Biosens. Bioelectron., 2013, 41, 551.

38. J. I. Gowda and S. T. Nandibewoor, Asian J. Pharm. Sci., 2014, $9,42$.

39. X. Shen, D. Liu, C. Zhu, Y. Li, Y. Liu, and T. You, Electrochem. Commun., 2020, 110, 106637.

40. J. D. Patterson, B. D. Debaryshe, and E. Ramsey, J. Am. Psychol., 1989, 44, 329.

41. J. L. Calais, Int. J. Quantum Chem., 1993, 47, 101.

42. T. Karthick and P. Tandon, J. Mol. Model., 2016, 22, 142. 